\title{
When Does the Set of $(a, b, c)$-Core Partitions Have a Unique Maximal Element?
}

\author{
Amol Aggarwal * \\ Department of Mathematics \\ Massachusetts Institute of Technology \\ Massachusetts, U.S.A. \\ agg_a@mit.edu
}

Submitted: Oct 21, 2014; Accepted: May 9, 2015; Published: May 22, 2015

Mathematics Subject Classifications: 05A17

\begin{abstract}
In 2007, Olsson and Stanton gave an explicit form for the largest $(a, b)$-core partition, for any relatively prime positive integers $a$ and $b$, and asked whether there exists an $(a, b)$-core that contains all other $(a, b)$-cores as subpartitions; this question was answered in the affirmative first by Vandehey and later by Fayers independently. In this paper we investigate a generalization of this question, which was originally posed by Fayers: for what triples of positive integers $(a, b, c)$ does there exist an $(a, b, c)$-core that contains all other $(a, b, c)$-cores as subpartitions? We completely answer this question when $a, b$, and $c$ are pairwise relatively prime; we then use this to generalize the result of Olsson and Stanton.
\end{abstract}

Keywords: Young diagram; hook length; core partition; numerical semigroup; UM-set; poset-UM

\section{Introduction}

A partition is a finite, nonincreasing sequence $\lambda=\left(\lambda_{1}, \lambda_{2}, \ldots, \lambda_{r}\right)$ of positive integers. The sum $\sum_{i=1}^{r} \lambda_{i}$ is the size of $\lambda$ and is denoted by $|\lambda|$; the integer $r$ is the length of $\lambda$. A partition $\mu=\left(\mu_{1}, \mu_{2}, \ldots, \mu_{s}\right)$ is a subpartition of $\lambda$ if $s \leqslant r$ and $\mu_{i} \leqslant \lambda_{i}$ for each integer $i \in[1, s]$; in this case, we say that $\mu \subseteq \lambda$.

We may represent $\lambda$ by a Young diagram, which is a collection of $r$ left-justified rows of cells with $\lambda_{i}$ cells in row $i$. The hook length of any cell $C$ in the Young diagram is defined to be the number of cells to the right of, below, or equal to $C$. For instance, Figure 1 shows the Young diagrams and hook lengths of the partitions $(6,4,2,2,1,1)$ and $(5,3,1,1)$.

\footnotetext{
*Supported by NSF Grant 1358659 and NSA Grant H98230-13-1-0273.
} 
Let $\beta(\lambda)$ denote the set of hook lengths in the leftmost column of the Young tableaux associated with $\lambda$; equivalently, $\beta(\lambda)=\left(\lambda_{1}+r-1, \lambda_{2}+r-2, \ldots, \lambda_{r}\right)$. For instance, Figure 1 shows that $\beta(6,4,2,2,1,1)=\{11,8,5,4,2,1\}$ and $\beta(5,3,1,1)=(8,5,2,1)$.

For any set of positive integers $A=\left\{a_{1}, a_{2}, \ldots, a_{k}\right\}$, a partition is an $A$-core if no cell of its Young diagram has hook length in $A$. Let the set of $A$-cores be $C(A)$; Figure 1 shows that $(6,4,2,2,1,1) \in C(3,7)$ and $(5,3,1,1) \in C(3,7,11)$.

Core partitions are known to be related to representations of the symmetric group; for instance, Olsson and Stanton use simultaneous core partitions in [12] to prove the NavarroWillems conjecture for symmetric groups. Core partitions are also known to be related to the alcove geometry for certain types of Coxeter groups (see $[4,8,9]$ ). Recently, there has been a growing interest in simultaneous core partitions because of their relationship with numerical semigroups (see $[1,2,4,15,17]$ ).

\begin{tabular}{|c|c|c|c|c|c|}
\hline 11 & 8 & 5 & 4 & 2 & 1 \\
\hline 8 & 5 & 2 & 1 & & \\
\hline 5 & 2 & & & & \\
\hline 4 & 1 & & & & \\
\hline 2 & & & & & \\
\hline 1 & & & & & \\
\hline
\end{tabular}

\begin{tabular}{|l|l|l|l|l|}
\hline 8 & 5 & 4 & 2 & 1 \\
\cline { 1 - 2 } 5 & 2 & 1 & \multicolumn{2}{|l}{} \\
\cline { 1 - 1 } 2 & \multicolumn{3}{|l}{} \\
\cline { 1 - 1 } 1 & \multicolumn{3}{|l}{} & \multicolumn{2}{|c}{} \\
\cline { 1 - 1 } & &
\end{tabular}

Figure 1: To the left is the Young diagram of $\kappa_{3,7}=(6,4,2,2,1,1)$ and to the right is the Young diagram of $(5,3,1,1)$; each cell contains its hook length.

During the past decade, combinatorialists have studied properties of $C(A)$ when $|A|=$ 2 (see $[1,3,4,5,6,8,9,10,11,12,15,16]$ ). For instance, Anderson showed that $|C(a, b)|=\left(\begin{array}{c}a+b \\ a\end{array}\right) /(a+b)$ if $a$ and $b$ are relatively prime; in particular, there are finitely many $(a, b)$-cores $[3]$.

This implies that there is an $(a, b)$-core of maximum size. Auckerman, Kane, and Sze conjectured in [5] that this size is $\left(a^{2}-1\right)\left(b^{2}-1\right) / 24$. This was verified in 2007 by Olsson and Stanton, who also found the core of this size explicitly in terms of $a$ and $b$ [12]. Specifically, they established the following result.

Theorem 1.1. For any relatively prime positive integers $a$ and $b$, there is a unique $(a, b)$ core $\kappa_{a, b}$ of maximum size; a positive integer is in $\beta\left(\kappa_{a, b}\right)$ if and only if it is of the form $a b-i a-j b$ for some positive integers $i$ and $j$.

Figure 1 depicts the Young diagram of $\kappa_{3,7}=(6,4,2,2,1,1)$. In their proof of Theorem 1.1, Stanton and Olsson showed that $\kappa_{n, n+1}$ contains every other $(n, n+1)$-core as a subpartition, for each integer $n \geqslant 2[12]$. They then asked whether $\kappa_{a, b}$ contains all other $(a, b)$-cores as subpartitions, for every pair of relatively prime positive integers $(a, b)$. Vandehey answered this question in the affirmative in 2009 through the use of abacus diagrams [16]. Recently, Fayers obtained the same result by analyzing actions of the affine symmetric group on the set of $a$-cores (and on the set of $b$-cores) [8]. 
To see an example of Vandehey's theorem, let $A=(3,5)$. The nonempty partitions in $C(A)$ are $\{(1),(2),(1,1),(3,1),(2,1,1),(4,2,1,1)\}$, and every element of $C(A)$ is contained in the $(3,5)$-core $(4,2,1,1)$. However, this containment phenomenon does not necessarily hold when $|A| \geqslant 3$ and $\operatorname{gcd} A=1$. For instance, if $A=\{3,4,5\}$, then the nonempty elements of $C(A)$ are $(1,1)$ and $(2)$; neither of these is contained in the other.

For any set of positive integers $A$, we say that $C(A)$ has a unique maximal element if there is an $A$-core $\kappa_{A}$ that contains every other $A$-core as a subpartition; in this case, the set $A$ is said to be $U M$. In [7], Fayers asked the following question.

Question 1.2. What triples of positive integers $(a, b, c)$ are UM?

Vandehey's result implies a partial result in this direction. For any set of positive integers $A$, let $S(A)$ be the numerical semigroup generated by $A$; equivalently, $A$ consists of all linear combinations of elements in $A$ with nonnegative integer coefficients. Due to the known fact that an $(a, b)$-core is an $(a+b)$-core (see [2], for instance), Vandehey's result implies that $(a, b, c)$ is $\mathrm{UM}$ if $a$ and $b$ are relatively prime and $c \in S(a, b)$. Recently, Yang, Zhong, and Zhou showed that $(2 k+1,2 k+2,2 k+3)$ is not UM for any positive integer $k$ [17].

In this paper we give a partial answer to Question 1.2. We call a triple of positive integers $(a, b, c)$ aprimitive if either $a \in S(b, c), b \in S(a, c)$, or $c \in S(a, b)$. The following theorem gives a restriction on triples that can be UM.

Theorem 1.3. Suppose that $(a, b, c)$ is a triple of positive integers such that $\operatorname{gcd}(a, b, c)=$ 1 ; let $p=\operatorname{gcd}(a, b), q=\operatorname{gcd}(a, c), r=\operatorname{gcd}(b, c)$, and $d, e, f$ be integers such that $(a, b, c)=(d p q, e p r, f q r)$ as ordered triples. If $(a, b, c)$ is $U M$, then $(d, e, f)$ is aprimitive.

Not all triples of the form given by the above theorem are UM; for instance, we will see in Section 2 that $(4,5,6)$ is not UM. However, we may use Theorem 1.3 to answer Question 1.2 completely when $a, b$, and $c$ are pairwise relatively prime.

Corollary 1.4. If $a<b<c$ are pairwise relatively prime positive integers, then $(a, b, c)$ is $U M$ if and only if $c \in S(a, b)$.

Proof. As noted previously, Vandehey's theorem implies that $(a, b, c)$ is UM if $c \in S(a, b)$. Setting $p=q=r=1$ in Theorem 1.3 yields the converse.

Corollary 1.4 can be viewed as a converse to Vandehey's theorem; it also generalizes the previously mentioned result of Yang, Zhong, and Zhou.

Using Theorem 1.3, we will also be able to express the unique maximal $(a, b, c)$-core $\kappa_{a, b, c}$ in terms of $a, b$, and $c$ if the triple $(a, b, c)$ is UM.

Theorem 1.5. Suppose that $A=(a, b, c)$ is a triple of positive integers that is UM; let $p=\operatorname{gcd}(a, b), q=\operatorname{gcd}(a, c), r=\operatorname{gcd}(b, c)$, and $d, e, f$ be integers such that $(a, b, c)=$ (dpq,epr, fqr) as ordered triples. If $f \in S(d, e)$, then a positive integer is in $\beta\left(\kappa_{A}\right)$ if and only if it is of the form $(d e+f) p q r-i a-j b-k c$ for some positive integers $i, j$, and $k$. 
Observe that letting $p=q=r=1$ in Theorem 1.5 yields Theorem 1.1 of Olsson and Stanton, due to Vandehey's theorem.

The proofs of Theorem 1.3 and Theorem 1.5 use a recently developed characterization of simultaneous cores through numerical semigroups, which we will explain further in the next section.

\section{Proofs of Theorems 1.3 and 1.5}

In this section, we first explain a bijection (originally due to Stanley and Zanello in [15] when $|A|=2$ and later generalized to arbitrary sets $A$ by Amdeberhan and Leven in [2]) between $A$-cores and order ideals of some poset $P(A)$. We will then use this bijection to obtain a preliminary necessary condition for a set $A$ to be UM. Using this condition, we will establish Theorem 1.3 and Theorem 1.5.

Now let us define the poset $P(A)$. The elements of $P(A)$ are those of $\mathbb{Z}_{\geqslant 0} \backslash S(A)$, the set of positive integers not contained in the numerical semigroup generated by $A$. Notice that if $\operatorname{gcd} A=1$, then $|P(A)|<\infty$; we will suppose that this is the case for the remainder of the section. The order on $P(A)$ is fixed by requiring $p \in P(A)$ to be greater than $q \in P(A)$ if $p-q \in S(A)$. Under this partial order, $P(A)$ is a poset; we will follow the poset terminology given in Chapter 3 of Stanley's text $[13,14]$. Figure 2 depicts the Hasse diagrams of the posets $P(3,7)$ and $P(3,7,11)$.
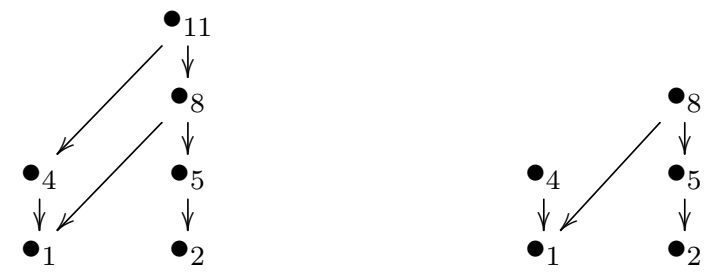

Figure 2: The Hasse diagrams of $P(3,7)$ and $P(3,7,11)$ are shown on the left and right, respectively.

The following lemma is due to Amdeberhan and Leven in [2].

Lemma 2.1. There is a bijection between $C(A)$ and the set of order ideals of $P(A)$. Specifically, for each partition $\lambda$, the set $\beta(\lambda)$ is an order ideal of $P(A)$ if and only if $\lambda$ is an A-core.

For instance, suppose that $A \subseteq\{3,7,11\}$; then, $(5,3,1,1)$ is an $A$-core and its beta set $\{8,5,2,1\}$ is an order ideal of $P(A)$. Furthermore, $\kappa_{3,7}=(6,4,2,2,1,1)$ is a $(3,7)$ core and its beta set $\{1,2,4,5,8,11\}$ is an order ideal of $P(3,7)$; however, $\kappa_{3,7}$ is not a $(3,7,11)$-core and its beta set is not an order ideal of $P(3,7,11)$.

From Lemma 2.1, there is an $A$-core $\kappa_{A}^{\prime}$ such that $\beta\left(\kappa_{A}^{\prime}\right)=P(A)$. The following result states that $\kappa_{A}^{\prime}$ is the unique maximal element of $C(A)$ if $A$ is $\mathrm{UM}$.

Corollary 2.2. If a set of positive integers $A$ is $U M$, then $\kappa_{A}^{\prime}=\kappa_{A}$. 
Proof. The bijection in Lemma 2.1 is length preserving; since the longest order ideal of $P(A)$ is $P(A)$, the longest $A$-core is $\kappa_{A}^{\prime}$. Therefore, $\kappa_{A}^{\prime}$ is not contained in any other $A$-core, which implies that $\kappa_{A}$ is the unique maximal element of $C(A)$ because $A$ is $\mathrm{UM}$. Thus, $\kappa_{A}^{\prime}=\kappa_{A}$.

Now, we call a poset $P$ poset- $U M$ if $P$ contains a unique maximal element. For instance, Figure 2 shows that $P(3,7)$ is poset-UM with unique maximal element 11 and that $P(3,7,11)$ is not poset-UM since it has both 4 and 8 as maximal elements. The following known lemma gives examples of posets that are poset-UM.

Lemma 2.3. Suppose that $a$ and $b$ are relatively prime positive integers; then, $P(a, b)$ is poset-UM with maximal element $a b-a-b$. Equivalently, a positive integer is in $P(a, b)$ if and only if it is of the form $a b-j a-h b$ for some integers $j \in[1, b-1]$ and $h \in[1, a-1]$.

The following proposition yields a preliminary necessary condition for a set of positive integers to be UM.

Proposition 2.4. If a set of positive integers $A$ is $U M$, then $P(A)$ is poset-UM.

Proof. Suppose that $P(A)$ is not poset-UM but that $A$ is UM. Consider the element $m \in P(A)$ of maximum magnitude; for instance, if $A=(3,7,11)$, then $m=8$. Since $P(A)$ is not poset-UM, there is an order ideal $I \subseteq P(A)$ containing $m$ but not equal to $P(A)$. By Lemma 2.1, there are $A$-cores $\lambda=\left(\lambda_{1}, \lambda_{2}, \ldots, \lambda_{r}\right)$ and $\kappa=\kappa_{A}=\left(\kappa_{1}, \kappa_{2}, \ldots, \kappa_{s}\right)$ such that $\beta(\lambda)=I$ and $\beta(\kappa)=P(A)$. By Corollary 2.2, $\kappa$ is the unique maximal element of $C(A)$; therefore, $\lambda \subset \kappa$. Hence, $m-r+1=\lambda_{1} \leqslant \kappa_{1}=m-s+1$, which is a contradiction since $s=|P(A)|>|I|=r$.

As an application, the above proposition implies that $(3,7,11)$ is not UM. Observe that the converse of Proposition 2.4 does not always hold. For instance, suppose that $A=\{4,5,6\}$; then, $P(A)=\{1,2,3,7\}$ is poset-UM with unique maximal element 7 . Therefore the longest $A$-core is $\kappa_{A}=(3,1,1,1)$, which does not contain the $A$-core $(2,2)$ as a subpartition.

We will now classify all triples of positive integers $(a, b, c)$ whose associated posets $P(a, b, c)$ are poset-UM. The following proposition forms a bijection between the maximal elements of $P(a, b, c)$ and the maximal elements of $P(d, e, f)$ for particular triples $(a, b, c)$ and $(d, e, f)$.

Proposition 2.5. Suppose that $(a, b, c)$ is a triple of positive integers with $\operatorname{gcd}(a, b, c)=1$; let $\operatorname{gcd}(a, b)=p, \operatorname{gcd}(a, c)=q, \operatorname{gcd}(b, c)=r$, and $d, e, f$ be integers such that $(a, b, c)=$ $(d p q, e p r, f q r)$ as ordered triples. For $m$ and $n$ positive integers, $(m-1) d+(n-1) e-f$ is a maximal element of $P(d, e, f)$ if and only if $(m r-1) d p q+(n q-1) e p r-f q r$ is a maximal element of $P(a, b, c)$.

Proof. Let $s=(m-1) d+(n-1) e-f$ and $t=(m r-1) d p q+(n q-1) e p r-f q r$. Suppose that $s$ is a maximal element of $P(d, e, f)$; we will show that $t$ is a maximal element of $P(a, b, c)$. Let us first verify that $t \in P(a, b, c)$. Suppose to the contrary that $t \in S(a, b, c)$. Then, 
there are nonnegative integers $h, i, j$ such that $(m r-1) d p q+(n q-1) e p r-f q r=h d p q+$ $i e p r+j f q r$. Since $\operatorname{gcd}(a, b, c)=1$, we have that $\operatorname{gcd}(r, p)=\operatorname{gcd}(r, q)=1=\operatorname{gcd}(r, d)$. The previous equality implies that $(m r-1-h) d p q$ is a multiple of $r$, which yields $h=r h^{\prime}-1$ for some positive integer $h^{\prime}$. Similarly, $i=q i^{\prime}-1$ for some positive integer $i^{\prime}$; therefore, $\left(m-h^{\prime}\right) d p+\left(n-i^{\prime}\right) e p=(j+1) f$. Thus, $j=j^{\prime} p-1$ for some positive integer $j^{\prime}$; hence $s=(m-1) d+(n-1) e-f=\left(h^{\prime}-1\right) d+\left(i^{\prime}-1\right) e+\left(j^{\prime}-1\right) f \in S(d, e, f)$, which is a contradiction.

Now, to see that $t$ is maximal, it suffices to verify that $t+a, t+b, t+c \in S(a, b, c)$. Observe that $t+a=m d p q r+(n q-1) e p r-f q r=p q r(s+d)+\operatorname{epr}(q-1)+f q r(p-1) \in$ $S(d p q, e p r, f q r)$ because $s+d \in S(d, e, f)$ by the maximality of $s$. By similar reasoning, $t+b \in S(a, b, c)$; since $t+c=(m r-1) d p q+(n q-1) e p r \in S(a, b, c)$, it follows that $t$ is a maximal element of $P(a, b, c)$.

This implies that $t$ is a maximal element of $P(a, b, c)$ if $s$ is a maximal element of $P(d, e, f)$. Through similar reasoning, one may show that $s$ is a maximal element of $P(d, e, f)$ if $t$ is a maximal element of $P(a, b, c)$.

The following corollary reduces the classification of triples $(a, b, c)$ whose associated posets $P(a, b, c)$ are poset-UM to the case when $a, b$, and $c$ are pairwise relatively prime.

Corollary 2.6. Suppose that $(a, b, c)$ is a triple of positive integers such that $\operatorname{gcd}(a, b, c)=$ $1 ;$ let $\operatorname{gcd}(a, b)=p, \operatorname{gcd}(a, c)=q, \operatorname{gcd}(b, c)=r$, and $d, e, f$ be integers such that $(a, b, c)=$ $(d p q, e p r, f q r)$ as ordered triples. Then, $P(a, b, c)$ is poset-UM if and only if $P(d, e, f)$ is poset-UM.

Proof. Suppose that $P(d, e, f)$ is not poset-UM and let $s_{1}$ and $s_{2}$ be two distinct maximal elements of $P(d, e, f)$. Since $s_{1}$ and $s_{2}$ are maximal, we have that $s_{1}+f, s_{2}+f \in S(d, e)$; thus, there are positive integers $m_{1}, n_{1}, m_{2}, n_{2}$ such that $s_{1}=\left(m_{1}-1\right) d+\left(n_{1}-1\right) e-f$ and $s_{2}=\left(m_{2}-1\right) d+\left(n_{2}-1\right) e-f$. Let $t_{1}=\left(r m_{1}-1\right) d p q+\left(q n_{1}-1\right) e p r-f q r$ and $t_{2}=\left(r m_{2}-1\right) d p q+\left(q n_{2}-1\right) e p r-f q r$; by Proposition $2.5, t_{1}$ and $t_{2}$ are maximal elements of $P(a, b, c)$. Since $s_{1}$ and $s_{2}$ are distinct, $t_{1} \neq t_{2}$; thus $P(a, b, c)$ has two distinct maximal elements and is therefore not poset-UM.

Now, suppose that $P(a, b, c)$ is not poset-UM and let $t_{1}$ and $t_{2}$ be two distinct maximal elements of $P(a, b, c)$. As above, there are positive integers $m_{1}^{\prime}, n_{1}^{\prime}, m_{2}^{\prime}, n_{2}^{\prime}$ such that $t_{1}=$ $\left(m_{1}^{\prime}-1\right) d p q+\left(n_{1}^{\prime}-1\right) e p r-f q r$ and $t_{2}=\left(m_{2}^{\prime}-1\right) d p q+\left(n_{2}^{\prime}-1\right) e p r-f q r$. We claim that $m_{1}^{\prime}$ is a multiple of $r$. Indeed, since $t_{1}$ is maximal, $m_{1}^{\prime} d p q+\left(n_{1}^{\prime}-1\right) e p r-f q r=t_{1}+a \in S(a, b, c)$; therefore, there are nonnegative integers $h, i, j$ such that $m_{1}^{\prime} d p q+\left(n_{1}^{\prime}-1\right)$ epr $-f q r=$ $h d p q+i e p r+j f q r$. Since $t_{1} \notin S(a, b, c)$, we have that $h=0$. Therefore, $r$ divides $m_{1}^{\prime} d p q$; the fact that $\operatorname{gcd}(d, r)=\operatorname{gcd}(p, r)=\operatorname{gcd}(q, r)=1$ thus yields $r$ divides $m_{1}^{\prime}$. Hence, there is an integer $m_{1}$ such that $m_{1}^{\prime}=m_{1} r$; by similar reasoning, there are integers $n_{1}, m_{2}, n_{2}$ such that $n_{1}^{\prime}=n_{1} q, m_{2}^{\prime}=m_{2} r$, and $n_{2}^{\prime}=n_{2} q$. By Proposition 2.5, $s_{1}=\left(m_{1}-1\right) d+\left(n_{1}-1\right) e-f$ and $s_{2}=\left(m_{2}-1\right) d+\left(n_{2}-1\right) e-f$ are unique maximal elements of $P(d, e, f)$. Since $t_{1}$ and $t_{2}$ are distinct, $s_{1} \neq s_{2}$; this implies that $P(d, e, f)$ has two maximal elements and is therefore not poset-UM.

The following proposition, which we will prove in the next section, classifies all triples of pairwise relatively prime positive integers $(a, b, c)$ such that $P(a, b, c)$ is poset-UM. 
Proposition 2.7. If $a<b<c$ are pairwise relatively prime positive integers, then $P(a, b, c)$ is poset-UM if and only if $c \in S(a, b)$.

Assuming Proposition 2.7, we may classify all triples of positive integers $(a, b, c)$ for which $P(a, b, c)$ is poset-UM.

Corollary 2.8. Suppose that $(a, b, c)$ is a triple of positive integers such that $\operatorname{gcd}(a, b, c)=$ 1; let $p=\operatorname{gcd}(a, b), q=\operatorname{gcd}(a, c), r=\operatorname{gcd}(b, c)$, and $d, e, f$ be integers such that $(a, b, c)=$ $(d p q, e p r, f q r)$ as ordered triples. Then, $P(a, b, c)$ is poset-UM if and only if $(d, e, f)$ is aprimitive.

Proof. This follows from Corollary 2.6 and Proposition 2.7.

We may now establish Theorem 1.3.

Proof of Theorem 1.3. This follows from Proposition 2.4 and Corollary 2.8.

Using Theorem 1.3, we may now establish Theorem 1.5.

Proof of Theorem 1.5. Let $f=m d+n e$ for some nonnegative integers $m$ and $n$. By Lemma 2.3, the unique maximal element of $P(d, e, f)$ is $d e-d-e=(d+n-1) e+(m-1) d-$ $f$. By Proposition 2.4, $P(A)$ has a unique maximal element; by Proposition 2.5, the unique maximal element of $P(A)$ is $(d e+m d+n e) p q r-d p q-e p r-f q r=(d e+f) p q r-a-b-c$. Hence, a positive integer is in $P(A)$ if and only if it is of the form $(d e+f) p q r-i a-j b-k c$ for some positive integers $i, j$, and $k$. This implies the corollary since $\beta\left(\kappa_{A}\right)=P(A)$.

\section{$3 \quad$ Proof of Proposition 2.7}

Throughout this section, we will adopt the notation given in Proposition 2.7. If $c \in S(a, b)$, then $P(a, b, c)=P(a, b)$ because $S(a, b, c)=S(a, b)$; therefore, the proposition follows from Lemma 2.3.

So, let us suppose that $c \notin S(a, b)$. By Lemma 2.3, there are positive integers $s_{1} \in$ $[1, b-1]$ and $t_{1} \in[1, a-1]$ such that $c=a b-s_{1} a-t_{1} b$. Let $k$ be the largest positive integer such that $i c \notin S(a, b)$ for each integer $i \in[1, k]$; observe that $k<b$.

By Lemma 2.3, there are integers $s_{1}, s_{2}, \ldots, s_{k} \in[1, b-1]$ and $t_{1}, t_{2}, \ldots, t_{k} \in[1, a-1]$ such that $i c=a b-s_{i} a-t_{i} b$ for each integer $i \in[1, k]$; moreover, let $\left(s_{0}, t_{0}\right)=(0, a)$ and $\left(s_{k+1}, t_{k+1}\right)=(b, 0)$. Observe that the $s_{i}$ are distinct, since we would otherwise have that $(j-i) c=\left(t_{i}-t_{j}\right) b$ for some integers $1 \leqslant i<j \leqslant k$, which contradicts the facts that $k<b$ and $\operatorname{gcd}(b, c)=1$; similarly, we see that the $t_{i}$ are distinct.

By establishing properties about the $s_{i}$ and $t_{i}$, we will show that $P(a, b, c)$ has at least two distinct maximal elements, which would imply that $P(a, b, c)$ is not UM. Let us begin with the following property.

Lemma 3.1. For all integers $i, j \in[0, k+1]$, we have that $t_{i}<t_{j}$ if and only if $s_{i}>s_{j}$. 
Proof. Suppose to the contrary that there exist integers $i, j \in[0, k+1]$ such that $t_{i}<t_{j}$ and $s_{i}<s_{j}$. Then, $i c=a b-s_{i} a-t_{i} b>a b-s_{j} a-t_{j} b=j c$, which implies that $i>j$. Furthermore, $i \neq k+1$ since $s_{k+1}=b \geqslant s_{h}$ for any integer $h \in[0, k+1]$. Therefore, $i-j \in[1, k]$, so $(i-j) c \notin S(a, b)$ by the definition of $k$. However, $(i-j) c=$ $\left(s_{j}-s_{i}\right) a+\left(t_{j}-t_{i}\right) b \in S(a, b)$, which is a contradiction. Therefore, the proposition holds.

Now, let $m, n \in[1, k]$ be integers such that $s_{m}=\min _{i \in[1, k]} s_{i}$ and $t_{n}=\min _{i \in[1, k]} t_{i}$. By Lemma 3.1, we have that $s_{n}=\max _{i \in[1, k]} s_{i}$ and $t_{m}=\max _{i \in[1, k]} t_{i}$.

Lemma 3.2. We have that $k=m+n-1$.

Proof. We will first show that $k<m+n$. Suppose otherwise, so in particular $2 a b-$ $\left(s_{m}+s_{n}\right) a-\left(t_{m}+t_{n}\right) b=(m+n) c \notin S(a, b)$. Thus $2 a b-\left(s_{m}+s_{n}\right) a-\left(t_{m}+t_{n}\right) b=$ $a b-s_{m+n} a-t_{m+n} b$, so $a b=\left(s_{m}+s_{n}-s_{m+n}\right) a+\left(t_{m}+t_{n}-t_{m+n}\right) b$. Since $\left|s_{m}+s_{n}-s_{m+n}\right|<2 b$, $\left|t_{m}+t_{n}-t_{m+n}\right|<2 a$, and $\operatorname{gcd}(a, b)=1$, this implies that either $s_{m}+s_{n}-s_{m+n}=b$ and $t_{m}+t_{n}=t_{m+n}$ or $s_{m}+s_{n}=s_{m+n}$ and $t_{m}+t_{n}-t_{m+n}=a$. Without loss of generality, suppose that the former holds; then $s_{m+n}=s_{m}+s_{n}-b<s_{m}$, which contradicts the minimality of $s_{m}$. Therefore, $k \leqslant m+n-1$.

To see that $k \geqslant m+n-1$, observe that $(m+n-i) c=a b-\left(s_{m}+s_{n}-s_{i}\right) a-\left(t_{m}+t_{n}-t_{i}\right) b$ for each integer $i \in[1, k]$. Since $0<s_{m} \leqslant s_{i} \leqslant s_{n}$ and $0<t_{n} \leqslant t_{i} \leqslant t_{m}$, we obtain that $(m+n-i) c$ is of the form $a b-a h-b j$ for some positive integers $h$ and $j$. Thus Lemma 2.3 implies that $i c \notin S(a, b)$ for each integer $i \in[1, m+n-1]$, which yields $k \geqslant m+n-1$. Hence, $k=m+n-1$.

Now let $\left\{r_{0}, r_{1}, \ldots, r_{k+1}\right\}$ be a permutation of $\{0,1,2, \ldots, k, k+1\}$ such that $s_{r_{0}}<s_{r_{1}}<$ $\cdots<s_{r_{k+1}}$. By Lemma 3.1, we have that $t_{r_{k}}<t_{r_{k-1}}<\cdots<t_{r_{1}}$. Furthermore, observe that $r_{0}=0 ; r_{k+1}=k+1 ; r_{1}=m$; and $r_{k}=n$.

Let $p_{i}=a b-\left(s_{r_{i}}+1\right) a-\left(t_{r_{i+1}}+1\right) b$ for each integer $i \in[0, k]$. Observe that the $p_{i}$ are positive since $p_{i} \geqslant a b-s_{r_{i}+1} a-t_{r_{i+1}} b-b=r_{i+1} c-b$, which is positive since $b<c$. We will show that $p_{j}$ and $p_{j+1}$ are both maximal elements of $P(a, b, c)$ for some integer $j \in[0, k]$. However, let us first show the following properties about $p_{i}$ for any integer $i \in[0, k]$.

Lemma 3.3. For any integer $i \in[0, k]$, we have that $p_{i} \in P(a, b, c)$ but $p_{i}+a, p_{i}+b \notin$ $P(a, b, c)$.

Proof. Let us first show that the former claim holds; suppose to the contrary that $p_{i} \in$ $S(a, b, c)$ for some integer $i \in[0, k]$. Then, there exist integers $f \in[0, b], h \in[0, a]$, and $j \in[0, k]$ such that $a b-\left(s_{r_{i}}+1\right) a-\left(t_{r_{i+1}}+1\right) b=p_{i}=f a+h b+j c=a b-\left(s_{j}-f\right) a-\left(t_{j}-h\right) b$. Thus $s_{j}>s_{r_{i}}$ and $t_{j}>t_{r_{i+1}}$. Letting $j=r_{h}$ for some integer $h \in[0, k+1]$, the former inequality implies that $h>i$ and the latter inequality implies that $h<i+1$; this is a contradiction, which yields $p_{i} \in P(a, b, c)$.

Now, for the second claim, observe that $p_{i}+a=a b-s_{r_{i}} a-\left(t_{r_{i+1}}+1\right) b=r_{i} c+\left(t_{r_{i}}-\right.$ $\left.t_{r_{i+1}}-1\right) b$, which is in $S(a, b, c)$ since $t_{r_{i}}>t_{r_{i+1}}$. Therefore, $p_{i}+a \notin P(a, b, c)$. By similar reasoning, $p_{i}+b \notin P(a, b, c)$. 
Let $k^{\prime}$ be such that $r_{k^{\prime}}=k$. We will show that $p_{j}$ and $p_{j+1}$ are distinct maximal elements of $P(a, b, c)$ with $j=k^{\prime}-1$. First, let us observe the following property about $k^{\prime}$.

Lemma 3.4. We have that $r_{k^{\prime}-1}=k-m$ and $r_{k^{\prime}+1}=k-n$.

Proof. We will only show the former statement since the proof of the latter is similar. Observe that, for any integer $i \in\left[0, k^{\prime}\right]$, we have that $a b-s_{k-r_{i}} a-t_{k-r_{i}} b=\left(k-r_{i}\right) c=$ $a b-\left(s_{r_{k}^{\prime}}-s_{r_{i}}\right) a-\left(a+t_{r_{k^{\prime}}}-t_{r_{i}}\right) b$. Since $s_{r_{i}}<s_{r_{k^{\prime}}}$, we have that $s_{k-r_{i}}=s_{r_{k^{\prime}}}-s_{r_{i}}$; this is minimal when $i=k^{\prime}-1$. Therefore, the minimality of $s_{m}$ implies that $r_{k^{\prime}-1}=k-m$.

Now we can establish the following lemma.

Lemma 3.5. We have that $p_{k^{\prime}-1}$ and $p_{k^{\prime}}$ are maximal elements of $P(a, b, c)$.

Proof. By Lemma 3.3, it suffices to show that $p_{k^{\prime}-1}+c \notin P(a, b, c)$ and that $p_{k^{\prime}}+c \notin$ $P(a, b, c)$. We will only show the first statement, since the proof of the second is similar.

Suppose to the contrary that $p_{k^{\prime}-1}+c \in P(a, b, c) \subset P(a, b)$. Then, Lemma 2.3 implies that there exist positive integers $j$ and $h$ such that $2 a b-\left(s_{r_{k^{\prime}-1}}+s_{1}+1\right) a-\left(t_{r_{k^{\prime}}}+t_{1}+1\right) b=$ $p_{k^{\prime}-1}+c=a b-j a-h b$. Using Lemma 3.4 and the fact that $r_{k^{\prime}}=k$, we find that $\left(s_{k-m}+s_{1}+1-j\right) a+\left(t_{k}+t_{1}+1-h\right) b=a b$. Therefore, we have that either $s_{k-m}+s_{1} \geqslant b$ or $t_{k}+t_{1} \geqslant a$.

By Lemma 3.2, we have that $k=m+n-1$. Thus, $a b-s_{k-m} a-t_{k-m} b=(k-m) c=$ $(n-1) c=a b-\left(s_{n}-s_{1}\right) a-\left(a+t_{n}-t_{1}\right) b$, which implies that $s_{k-m}+s_{1}=s_{n}<b$. Hence, $t_{k}+t_{1} \geqslant a$.

We claim that $t_{k}+t_{1} \leqslant a$ holds as well. Indeed, applying Lemma 3.2 again, we find that $a b-s_{k} a-t_{k} b=k c=(m+n-1) c=a b-\left(s_{m}+s_{n}-s_{1}\right) a-\left(t_{m}+t_{n}-t_{1}\right) b$. Thus, $s_{k}=s_{m}+s_{n}-s_{1}$ and $t_{k}=t_{m}+t_{n}-t_{1}$. Moreover, since $a b-\left(s_{m}+s_{n}\right) a-\left(t_{m}+t_{n}-a\right) b=$ $(m+n) c \in S(a, b)$, we have that $t_{k}+t_{1}=t_{m}+t_{n} \leqslant a$ by Lemma 2.3.

Therefore, $t_{m}+t_{n}=t_{k}+t_{1}=a$ and hence $a\left(b-s_{m}-s_{n}\right)=(m+n) c$. Since $a$ and $c$ are relatively prime, $b-s_{m}-s_{n}$ is a positive multiple of $c$; this contradicts the fact that $b<c$. Thus, $p_{k^{\prime}-1}+c \notin P(a, b, c)$.

Using Lemma 3.5, we may establish Proposition 2.7.

Proof of Proposition 2.7. If $c \in S(a, b)$, then $P(a, b, c)=P(a, b)$ because $S(a, b, c)=$ $S(a, b)$; therefore, the proposition follows from Lemma 2.3. If $c \notin S(a, b)$, then $P(a, b, c)$ has two distinct maximal elements by Lemma 3.5 , which implies that $P(a, b, c)$ cannot be UM. Therefore, $P(a, b, c)$ is $\mathrm{UM}$ if and only if $c \in S(a, b)$.

\section{Acknowledgements}

This research was conducted under the supervision of Joe Gallian at the University of Minnesota Duluth REU, funded by NSF Grant 1358659 and NSA Grant H98230-13-1-0273. The author heartily thanks Matt Fayers, Rishi Nath, and Joe Gallian for suggesting the topic of this project and for their valuable advice; the author also thanks David Moulton for his insightful discussions and Aaron Abrams and the referees for their suggestions. 


\section{References}

[1] A. Aggarwal. Armstrong's conjecture for $(k, m k+1)$-core partitions. European J. Combin., 47:54-67, 2015.

[2] T. Amdeberhan and E. Leven. Multi-cores, posets, and lattice paths. Preprint, 2014. arXiv: 1406.2250v2

[3] J. Anderson. Partitions which are simultaneously $t_{1^{-}}$and $t_{2}$-core. Discrete Math., 248:237-243, 2002.

[4] D. Armstrong, C. Hanusa, and B. Jones. Results and conjectures on simultaneous core partitions. European J. Combin., 41:205-220, 2014.

[5] D. Auckerman, B. Kane, and L. Sze. On simultaneous s-cores/t-cores. Discrete Math., 309:2712-2720, 2009.

[6] W. Chen, H. Huang, and L. Wang. Average size of a self-conjugate $(s, t)$-core partition.

Preprint, 2014. arXiv:1405.2175v1

[7] M. Fayers, Personal communication.

[8] M. Fayers. The $t$-core of an s-core. J. Combin. Theory Ser. A, 118:1525-1539, 2011.

[9] S. Fishel and M. Vazirani. A bijection between dominant Shi regions and core partitions. European J. Combin., 31:2087-2101, 2010.

[10] P. Johnson. Lattice points and simultaneous core partitions. Preprint, 2015. arXiv:1502.07934v1

[11] J. Olsson. A theorem on the cores of partitions. J. Combin. Theory Ser. A, 116:733$740,2009$.

[12] J. Olsson and D. Stanton. Block inclusions and cores of partitions. Aequationes Math., 74:90-110, 2007.

[13] R. Stanley. Enumerative Combinatorics. Volume 1. Second edition. Cambridge Studies in Advanced Mathematics, 49. Cambridge University Press, Cambridge, 2012.

[14] R. Stanley. Enumerative Combinatorics. Volume 2. Cambridge Studies in Advanced Mathematics, 62. Cambridge University Press, Cambridge, 1999.

[15] R. Stanley and F. Zanello. The Catalan case of Armstrong's conjecture on simultaneous core partitions. SIAM J. Discrete Math., 29:658-666, 2015.

[16] J. Vandehey. Containment in $(s, t)$-core partitions. Preprint, 2008. arXiv:0809.2134

[17] J. Yang, M. Zhong, and R. Zhou. On the enumeration of $(s, s+1, s+2)$-core partitions. Preprint, 2014. arXiv:1406.2583v1 